\title{
Depression in China: Integrating Developmental Psychopathology and Cultural-Clinical Psychology
}

\author{
Andrew G. Ryder ${ }^{1,2}$, Jiahong Sun ${ }^{1}$, Xiongzhao Zhu ${ }^{3}$, Shuqiao $\mathrm{Yao}^{3}$, and Yulia E. Chentsova-Dutton ${ }^{4}$ \\ ${ }^{1}$ Department of Psychology, Concordia University \\ ${ }^{2}$ Culture and Mental Health Unit, SMBD-Jewish General Hospital \\ ${ }^{3}$ Medical Research Center, Central South University \\ ${ }^{4}$ Department of Psychology, Georgetown University
}

\begin{abstract}
With a starting point in John Abela's groundbreaking developmental psychopathology research on adolescent depression in China, we aimed to review the state of the literature on Chinese depression across the lifespan. We began with Dr. Abela's published studies relevant to depression in China and our own research with adults before turning to the reference lists of these articles to find additional sources. Then we conducted literature searches using PsycINFO and PubMed to find other relevant studies published between April 2001 and April 2011. There are two distinct literatures on depression in China. Developmental psychopathology research has emphasized adolescent samples and cognitive models of causation; cultural-clinical psychology and cultural psychiatry research have emphasized adult samples and the meanings associated with emotions, symptoms, and syndromes. Both approaches to the study of depression in China have yielded important findings but have also highlighted issues that could be better addressed by incorporating the other approach. Beyond depression in China, the psychological study of culture and mental health more generally would benefit from greater exchange between developmental psychopathology and cultural-clinical psychology.
\end{abstract}

Two scientists are studying a population of fish swim ming downstream. The first walks along the riverbank, following a particular group of fish from the spawning grounds out to the sea. The second finds a choice vantage point; learns as much as possible about water quality, riverbed, and local flora; and observes the passing fish, affecting and affected by this local environment. Both scientists learn much that is relevant to understanding these fish, but both are missing a lot as well. Simply exchanging what they have learned would help, but would not complete the picture either-certain patterns are apparent only when attending both to the journey along the river and the local details at each point. Each scientist is concerned primarily with what they can learn from their own familiar approach, and they occasionally pass each other with a quick acknowledgment.

Preparation of this article was supported by a New Investigator Award from the Canadian Institutes of Health Research to Andrew G. Ryder.

Correspondence should be addressed to Andrew G. Ryder, Department of Psychology, Concordia University, 7141, rue Sherbrooke O. (PY153-2), Montréal, Québec, Canada H4B 1R6. E-mail: andrew. ryder@concordia.ca
Research on depression in Chinese populations shows a similar pattern. Our task was to review the innovative developmental psychopathology work conducted by John Abela and his colleagues in China over the past decade and place it in a larger context. This larger context is research from cultural psychiatry and, more recently, cultural-clinical psychology traditions. We quickly realized that we were engaging with two very different literatures; moreover, we kept observing that conceptual and methodological strengths of one approach are often all but unknown to the other. As a result, our goals for this article underwent their own developmental transformation over the course of writing. In addition to reviewing the state of the literature on depression in Chinese populations, we hope to open a dialogue between developmental psychopathology and cultural-clinical psychology. Doing so inevitably requires a critique of developmental psychopathology contributions. Although culture is not absent from this work, insufficient attention is paid to cultural context, especially methodologically. At the same time, a parallel critique of our own work in cultural-clinical psychology is also necessary. Although culture plays a minor role in the adolescent-focused developmental psychopathology research reviewed here, development is almost entirely absent from adult-focused cultural-clinical psychology research on depression in Chinese populations.

We begin with a brief review of some general issues in the study of depression in Chinese populations before turn- 
ing to the first of two major sections, on developmental psychopathology research. The key questions here focus on ways in which vulnerability and stress interact to predict the emergence of depression in adolescence. The second major section, on cultural-clinical psychology research, involves a different set of questions and a focus on adult populations. Here, the key questions are about symptom presentation and emotional expression, especially in depressed patients, and about the explanatory models held in the community. We close with some proposals for a specific integration of these findings and then speculate on the potential benefits of an ongoing dialogue between developmental psychopathology and cultural-clinical psychology across a range of mental health phenomena.

\section{Depression in China}

How is depression best defined? One difficulty is that "depression" can describe a mood state (depressed mood), a constellation of symptoms (a Major Depressive Episode), or a categorical diagnosis (Major Depressive Disorder). Measures of depression are replete with psychological and somatic symptoms, only a few of which relate directly to depressed mood, or indeed to any kind of mood (Bagby, Ryder, Schuller, \& Marshall, 2004). Proper attention to developmental and cultural perspectives makes an acceptable working definition of "depression" even more elusive. How depression is experienced, expressed, understood, and perceived by others is powerfully shaped by specific contexts (Kir mayer, 20011; Ryder, Ban, \& Dere, in press), contexts that in turn are shaped by the developmental niche one inhabits within a particular meaning system. As with emotions in general, the expression of dysphoric affect is governed by cultural expectations as to the salience of particular subjective experiences, the particular meanings attached to these experiences, the use of particular descriptive metaphors to convey them to others, and the perceived appropriateness of particular behavioral responses in particular situations (Ryder, Ban, \& Chentsova-Dutton, 2011).

This emphasis on particularity dovetails with the concerns of developmental psychology, in which people, especially young people, both shape and are shaped by situations over time. For example, children and adolescents acquire new skills as developing brains become capable of more complex tasks, building on skills already achieved; at the same time, this acquisition requires that learning take place in appropriate contexts (e.g., Ellis, Shirtcliff, Boyce, Deardorff, \& Essex, 2011). Similarly, in developmental psychopathology, disruptions in normal development can have very different effects depending on how they occur, and when (e.g., Cicchetti, Rogosch, Gunnar, \& Toth, 2010; Hankin, Badanes, Abela, \& Watamura, 2010). Depression-symptom, episode, or "disease"-unfolds over time.

The key challenge, therefore, when integrating develop- mental and cultural perspectives is that both the person and their contexts are revealed as moving, changing targets. It is doubtful that any country has exemplified rapid social change more clearly in recent years than the People's Republic of China (PRC). Over the past three decades, the PRC has undergone enormous economic, political, and sociocultural transformations, a process that has only accelerated in the past few years. Accompanying this widespread modernization, rapid urbanization, and increasing integration into a globalized world are changes in the views of mental health and illness, with consequences for depression.

Most striking, there is evidence that rates of depression have increased over this same period. Very low rates of depression in older studies were a puzzle for many years. Large mental health surveys conducted in the PRC showed an increase in rates between 1982 and 1993, although in both cases the rates were almost unbelievably low, with lifetimeand point-prevalence estimates in 1993 of $0.08 \%$ and $0.05 \%$, respectively (see Parker, Cheah, \& Roy, 2001). Such findings put the community rates in China at several hundred times lower than in North America. The World Health Organization's Global Burden of Disease project reported that unipolar major depression was the second largest contributor to disease burden in China (Murray \& López, 1996). Nevertheless, 1-year incidence rates were $2.3 \%$ for unipolar depression, in contrast to the $10.3 \%$ rate found in the United States (Kessler et al., 1994). National community surveys in Taiwan also identified low depression rates compared with other countries (Hwu, Yeh, \& Chang, 1989; Weissman et al., 1996). More recent epidemiological work in the PRC, by contrast, has found a $9 \%$ prevalence rate in the general population (Zhou, Zhang, Jiang, \& Wang, 2000) and estimates now range from $15 \%$ to $30 \%$ for Chinese adolescents (Hesketh \& Ding, 2005; Liu, Ma, Kurita, \& Tang, 1999; Wu, 2006). Whether these apparent increases are due to better recognition, greater willingness to endorse depressive symptoms, or actual increases in depression remains poorly understood.

Possible reasons underlying these changes point to fascinating questions about how supposedly universal constructs like "depression" can be powerfully shaped by historical forces, and we return to some of these issues at the close of this article. In the meantime, and regardless of specific reasons, increasing rates of depression have been accompanied by a much greater attention to depression and other forms of psychopathology in Chinese society. Two major themes have emerged, each targeting an aspect of depression's complexity, developmental or cultural. Driven in part by concern about adolescent depression in the PRC, several research groups have focused on the developmental pathways that lead to depression in Chinese contexts, emphasizing psychosocial and cognitive risk factors. Meanwhile, several more research groups have focused instead on the 
cultural context of adult depression in China and Chinese populations, emphasizing emotional and somatic symptom presentations.

Prominent among the first group of scholars was John Abela, a clinical psychologist and developmental psychopathologist who integrated his research interests in cognitive vulnerability with his intellectual curiosity about the Chinese cultural context (Gibb \& Hankin, 2010). Abela carried out an ambitious research program in the PRC designed to investigate the extent to which existing cognitive models of vulnerability to depression can be tested both crosssectionally and longitudinally in Chinese contexts and applied to understanding the burgeoning rates of depression in Chinese adolescents. It is to this work that we now turn.

\section{Developmental Psychopathology and Depression in Chinese Adolescents}

There has been long-standing interest in the interactional effects of stress and vulnerability on risk for depression. $\mathrm{Nu}$ merous studies have highlighted the role of cognitive factors such as rumination and low perceived control, along with psychosocial factors such as social support and coping style, in predicting depressive symptoms in adolescents (e.g., Connor-Smith \& Compas, 2004; Margaro \& Weisz, 2006; Park, Goodyear, \& Teasdale, 2004). Moreover, a prospective multiwave study found that cognitive vulnerabilities interact with stressors in predicting future levels of depression (Hankin, 2008). "To understand what may cause depression in youth, a vulnerability-stress framework, in which recent stressful events trigger an underlying predisposition, may be one of the most promising approaches" (Hankin, 2006, p. 105).

\section{Stress Vulnerability Hypothesis}

We begin by considering evidence for the stress- depression association and for cognitive vulnerability factors in Chinese adolescent samples. Cross-sectional and prospective studies have confirmed the link between stress and the onset of depression; moreover, psychosocial variables such as social support and coping are associated with depressive symptoms and also moderate the relation between stress and depression (Chen, Rubin, \& Li, 1995; Cheng, 1998; Hwang \& Myers, 2007; Ng \& Hurry, 2011; Sun, Tao, Hao, \& Wan, 2010). One of the few cross-national studies conducted in this area demonstrated similar associations between stressful life events and depression in Chinese and American adolescents (Greenberger, Chen, Tally, \& Dong, 2000). Research has identified cognitive symptoms of depression, such as hopelessness, at similar degrees of frequency in Hong Kong and American adolescents (Stewart et al., 2004; Stewart et al., 2002), and established links between cognitive distortions and internalizing problems (Leung \& Wong, 1998).
What about vulnerability? The response styles theory of depression posits that people will experience more severe depressive symptoms, and for longer durations, if they tend to use a ruminative coping response (e.g., Flynn, Kecmanovic, \& Alloy, 2010; Nolen-Hoeksema, 2000). Such a response involves a tendency to repetitively focus on the causes and consequences of depressive symptoms, dampening the ability to engage in effective and concentrated problem solving. In a retrospective evaluation of the response styles theory in a Chinese adolescent sample, higher levels of rumination were associated with greater likelihood of current or past Major Depressive Episode (MDE) after controlling for Neuroticism (Hong et al., 2010). These findings speak not only to the poten tial cross-cultural applicability of studying vulnerability factors but also to the clinical utility of identifying and targeting maladaptive coping responses when treating depressed Chinese adolescents.

In an effort to further understand the relation between coping style and depression, Abela and colleagues developed the Chinese version of the Responses to Stress Questionnaire. This questionnaire was then administered to a large sample of middle school students from Hunan, China (Xiao et al., 2010). In addition to supporting the psychometric properties of the instrument, the researchers found that lower levels of engagement coping at Time 1 predicted higher levels of depression at Time 2. A stress vulnerability model investigating the role of maladaptive coping strategies on the relation between stress and depression was then tested in samples of Canadian and Chinese adolescents (Auerbach, Abela, Zhu, $\&$ Yao, 2010). The model was supported in both samples: Participants who used a greater proportion of maladaptive coping strategies assessed at Time 1 experienced a greater increase in levels of depression following the occurrence of negative life events.

Abela and colleagues have also used prospective designs with multiple time points to study the impact of stress on subsequent depression in China. Yang and colleagues (2010) examined the impact of social support on subthreshold depressive symptoms following negative events. Depressive symptoms and social support were assessed at baseline, and a subsample of participants who exhibited subthreshold depressive symptoms was retained for these analyses. Over the subsequent 15 months, lower levels of peer support were associated with greater increase in depressive symptoms following negative life events, indicating the important moderating effect of social support on depression in response to stressors. Abela and colleagues (2011) used a prospective design to study the cognitive vulnerability factors posited by Abramson, Metalsky, and Alloy (1989) and Beck (1983) in Chinese adolescents. Over a 6-month period, both sets of cognitive vulnerability factors were associated with greater increase in depressive symptoms, but not anxious symptoms, following negative life events. Together, findings from these 
studies support the utility of studying vulnerability factors in Chinese adolescent depression from a stress vulnerability perspective.

\section{Stress Generation Hypothesis}

In contrast to the stress-vulnerability hypothesis where underlying vulnerability is activated in response to stressful events and thereby contributes to depression, the stress generation hypothesis posits that vulnerability factors actively contribute to the experience of stressful life events among people with a history of depression (Hammen, 1991).

This perspective involves a more agen tic role for the person and a stronger influence of the social environment on the onset and course of symptoms. Researchers have demonstrated that vulnerability factors predict subsequent stress elevations after controlling for baseline depressive symptoms, supporting the stress generation hypothesis (e.g., Eberhart \& Hammen, 2009).

This hypothesis also helps to explain the development of depression in Chinese adolescents. Starrs and colleagues (2010) adopted the UCLA Child Episodic Life Stress Interview (Rudolph \& Hammen, 1999) to assess negative life events in eight life domains using a cross-sectional design in a large sample of Chinese adolescents. Higher levels of interpersonal and cognitive vulnerabilities were associated with greater likelihood of reporting dependent negative events, after controlling for current and lifetime depression. Auerbach, Eberhart, and Abela (2010) then tested a mediation model of depression positing that people with a certain cognitive vulnerability actively contribute to the occurrence of life stressors that, in turn, lead to higher levels of depression. They used a time-lagged approach to examine the effect of cognitive vulnerability - specifically, low perceived control-test ing both stress vulnerability and stress generation models in samples of Canadian and Chinese adolescents. Both models were supported in Canadian adolescents, but only the stress generation model was supported in Chinese adolescents. Such findings raise questions about how cultural context shapes cognitive vulnerability.

\section{Cultural Issues Raised by John Abela's Research in China}

Despite rapid modernization and increasing engagement with a globalized world, Chinese adolescents inhabit a social milieu that differs markedly from their "Western" peers. For example, the oft-observed focus on education is reflected in research showing that interpersonal conflicts are the primary stressors for "Western" adolescents (Grant et al., 2006), whereas poor academic performance prospectively predicts higher levels of depression in Chinese children as young as 8 years of age (Chen et al., 1995). Although poor academic performance is associated with depression in both American and Chinese adolescents, there is a significantly stronger association in the latter group (Greenberger et al., 2000; see also Li \& Zhang, 2008). In addition, poor academic performance predicts suicidal ideation in Chinese adolescent samples (Hesketh, Ding, \& Jenkins, 2002). Even when a given stressor has an impact in many contexts, culture nonetheless shapes its meaning and assigned importance.

Auerbach, Eberhart, et al. (2010) made a similar case to explain why stress generation effects washed out vulnerability-stress effects in elucidating the role of low perceived control on stress and depression in Chinese adolescents. The authors proposed that in stressful times, Chinese adolescents with a diminished sense of control tend to contribute to the onset of subsequent stressors instead of reacting to existing stressful events, leading to higher depression levels. They noted that the intense pressures for university admission, especially given the relative lack of places, contributes to a sense among these adolescents that they do not have control over major aspects of their lives. Moreover, there is evidence from the cultural psychology literature to suggest that culture shapes the need for control and the importance of primary control (Sastry \& Ross, 1998; Weisz, Rothbaum, \& Blackburn, 1984). Primary control may be gaining in importance among Chinese adolescents under the growing influence of individualistic values (Auerbach, Eberhart, et al., 2010). These findings point to a line of inquiry combining developmental psychopathology and cultural-clinical psychology perspectives. Such studies should examine directly how perceived control is conceptualized in Chinese studies and the extent to which it can be used to better unpack cultural variation.

Although one aim of this research program was to understand sex differences in adolescent depression and in the role of cognitive vulnerabilities in predicting these differences, there has been a consistent lack of support for the expected sex differences in Chinese samples. Neither rates of subthreshold depression nor levels of rumination differed between boys and girls (Hong et al., 2010; Yang et al., 2010). Moreover, sex neither moderated nor mediated the association between vulnerability fac tors and either depression or stress generation (Auerbach, Abela, et al., 2010; Auerbach, Eberhart, et al., 2010; Starrs et al., 2010). Studies on adolescents in "Western" cultural contexts, by contrast, show a consistent tendency for female individuals to have earlier onset and more severe symptoms (e.g., Hankin, 2006).

Indeed, other studies on Chinese adolescent depression have replicated these balanced sex ratios with some studies even reporting higher levels of depression in boys. One study found that sex significantly predicted anxiety but not depression in Chinese adolescents (Li \& Zhang, 2008). In a largescale study of more than 2,000 Chinese adolescents, overall depression scores did not differ by sex, and by age 17 young men reported significantly higher levels (Liu et al., 1999). Fi- 
nally, a cross-national study found a greater sex difference in depression levels among American versus Chinese ado lescents (Greenberger et al., 2000). This consistent finding, different from what has been repeatedly observed in "Western" contexts, points to a second line of inquiry combining developmental psychopathology and cultural-clinical psychology perspectives. Such studies would begin by proposing specific a priori hypotheses for when sex differences are and are not observed, and then explicitly test them. In so doing, we would move beyond cataloguing an intriguing variation to understanding why it is observed.

\section{Cultural-Clinical Psychology and Depression in Chinese Adults}

Turning to the literature on Chinese depression that has taken an explicitly cultural perspective, we observe not only a strong tendency to focus on adults, but also that different kinds of questions entirely tend to get posed. In an attempt to understand the low rates of depression that were reported in China, researchers looked more closely at the potential role of symptom presentation-specifically, the oft-discussed tendencies for Chinese patients to emphasize somatic symptoms of depression and to minimize displays of emotional distress. We begin with a brief overview of our perspective on cultural clinical psychology. Then we examine research on depressive symptom presentation, emotional expression, and explanatory models before returning to the issue of historical change over time.

\section{Cultural-Clinical Psychology}

Let us first take a closer look at what we mean by culturalclinical psychology. Ryder and colleagues (2011) argued recently that a new field emerges at the intersection of cultural psychology and clinical psychology, and they described the implications in more detail elsewhere. Among other contributions, the infusion of a cultural psychology perspective into clinical research helps move us away from group differences to understanding how culture shapes psychological variation (Betancourt \& López, 1993; Heine \& Norenzayan, 2006; Markus \& Kitayama, 1991). Differentiating culture from "cultural group" highlights how individual variation exists within a cultural context-indeed, as an integral part of culture. A person in a group is not a perfect representative of that group but rather represents one of countless ways that a person in that group can be shaped by specific aspects of their cultural context.

Culture is best understood as meanings and practices, and especially as meaningful practices (Bruner, 1990). People who share a cultural context do not think and behave the same way but rather have a certain degree of intersubjective understanding of what is normative, expected, permitted, tolerated, or proscribed (Chiu, Gelfand, Yamagishi, Shteynberg, \& Wan, 2010). The idea of cultural scripts can be used to bridge meaning and practice (Ryder et al., 2011). These scripts refer to organized units of culturally salient knowledge that serve as mechanisms that allow for rapid, automatic retrieval and use of information. Once enacted, they are observable by others as behavior and become elements of the larger cultural context (DiMaggio, 1997; Ryder et al., 2011). As a result, they can in turn modify the meanings and hence the practices held by others in their context (Kashima, 2000), an example of what cultural psychologists call "mutual constitution."

Indeed, the core claim of cultural psychology is that human culture and human psychology are each grounded in the other: In Shweder's (1991) memorable phrase, that culture and mind "make each other up." We have proposed that the brain should be added to this conception, so that mutual constitution involves a single system with three levels (Kitayama \& Uskul, 2011; Ryder et al., 2011). Clinical psychologists and developmental psychopathologists have proposed increasingly sophisticated models of mental health that emphasize the central role of the brain and the ways in which it is shaped by genetic and, in turn, by evolutionary processes. Cultural neuroscientists, meanwhile, are documenting ways in which the brain is shaped by cultural context (Chiao, 2009), demonstrating that the human brain appears to be quite specifically adapted for cultural acquisition (Richerson \& Boyd, 2005; Wexler, 2006).

Given that culture-mind-brain constitutes a single system, changes at one level are expected to cascade through the whole system. It does not follow, however, that disorder at a higher level is caused by disorder at a lower level. Disorder can emerge from feedback loops in which the response to a problem exacerbates the problem, even when all the components of the loop are working normally (Kirmayer, 2007, 2012; Ryder et al., 2011; Ryder \& Chentsova-Dutton, in press). Rather than assuming that one can find the "real disorder" at the lowest level, problematic loops are best described at whatever level or levels provide the most explanatory power, which may differ depending on the purpose of the explanation. A necessarily limited causal story might best be told at one level, but the most effective clinical intervention might take place at a different level.

\section{Somatization and Psychologization}

Discussions of somatization and "psychologization" of distress involve a similar concern with multiple levels of analysis but with a tendency to privilege some levels over others. Psychoanalysts introduced the term "somatization" to refer to a defense mechanism in which anxious affect is allowed to reach consciousness only through bodily expression (Craig \& Boardman, 1990). This view implies that something is being somatized, namely, some kind of psychological experience. From a "Western" point of view, use of such a defense was interpreted as a sign of psychologi- 
cal immaturity, a legacy of mind- body dualism, in which central features of selfhood are located in the mind (Cheung, 1984; Draguns, 1996). In other words, the self might have a body but it is a mind (Ryder \& Chentsova-Dutton, in press). Traditional Chinese medicine, in contrast, views mind and body as integrated with one another as well as with social context; psychological and somatic experiences result from the intertwining of mind and body, with no privileging of one over the other (Cheung, 1995).

Kleinman (1982) conducted the first thorough study on this topic, using both psychiatric and ethnographic methods to assess "neurasthenia" patients in China. Neurasthenia-shenjing shuairuo-is a syndrome characterized primarily by fatigue and sleep problems, although it also includes mild emotional symptoms. Although he concluded that a large majority of these patients could be diagnosed with some form of depression, he argued that neurasthenia constituted a Chinese-specific way of presenting it. For example, most of these patients endorsed somatic symptoms as chief complaints, whereas only nine patients endorsed depressed mood. Nonetheless, descriptions of depression in China include psychological symptoms and somatic symptoms of depression are integral to "Western" understandings. We therefore must move beyond the stereotype of Chinese patients presenting only somatic symptoms-or "Western" patients presenting only psychological symptoms.

Almost two decades passed before any research directly compared Chinese and "Western" samples. Yen, Robins, and Lin (2000) first found that Chinese students seeking counselling reported considerably more somatic symptoms and fewer psychological symptoms on a questionnaire as compared with other Chinese students. Their second study then demonstrated that Chinese students reported significantly fewer somatic symptoms compared with Chinese-Americans and Euro-American students. The authors interpreted this pattern as suggesting that somatic symptom presentation in Chinese help-seekers is a means of accessing the health care system in China. None of these earlier studies involved direct empirical comparisons with clinical patients, however.

In the first such study, Parker et al. (2001) compared Chinese Malaysian and Euro-Australian outpatients. Participants completed a symptom questionnaire designed by the authors to capture symptoms of depression commonly reported in both cultural contexts. Chinese items were constructed in consultation with Chinese Malaysian mental health professionals and "Western" items were constructed by consulting common English-language measures of depression. A substantial majority of Chinese Malaysian patients nominated a somatic symptom as their first-ranked presenting complaint, as compared with a small minority of Euro-Australian patients. They were somewhat likely to endorse more somatic items across the questionnaire but had a much stronger tendency to endorse fewer psychological items.

Ryder and colleagues (2008) followed up these studies with the addition of clinical and structured interview methods in Chinese and Euro-Canadian psychiatric outpatients. The structured interview combined Diagnostic and Statistical Manual of Mental Disorders (4th ed., text rev.; American Psychiatric Association, 2000), international, and Chinesespecific diagnostic criteria for depression and neurasthenia, whereas the questionnaire included instruments originally developed in Chinese along with those originally developed in English. As anticipated, Chinese depressed outpatients were significantly more likely to endorse somatic symptoms and significantly less likely to endorse psychological symptoms. Similar to Parker and colleagues (2001), the latter effect was stronger. In addition, an externally oriented thinking style, or tendency to focus on the pragmatic details of everyday life rather than internal emotional states, mediated the relation between cultural group and somatic symptom emphasis.

A devaluation of emotional life does not fit well with North American cultural scripts, and indeed the measure of externally oriented thinking was a subscale of a questionnaire designed to measure emotional pathology. This measure might capture pathology in a cultural context that fosters ideals of emotional expression but may also represent an acceptable or even a healthy orientation in contexts with different norms about emotions (Dere, Falk, \& Ryder, 2012; Dion, 1996; Kirmayer, 1987). In keeping with this view, Dere and colleagues (2012) found that Chinese Canadian and EuroCanadian group differences in externally oriented thinking were mediated by degree of adherence to "Western" values. The relation between thinking style and cultural values has since been replicated in a sample of psychiatric outpatients in China (Dere et al., 2011). Cultural values point to scripts that shape the ways in which people attend to emotional (and other) cues, with implications for symptom presentation (Ryder \& Chentsova-Dutton, in press). At the same time, as we see, depression can also represent a failure to conform to normative cultural scripts about emotions, again with implications for the experience and expression of distress.

\section{The Cultural Norm Hypothesis}

Although psychological symptoms of major depression tap deficits in cognition, motivation, and self-image, the key symptoms of the Western-based definition of major depressive episode are emotional. There is ample evidence that depressed Euro-Americans show less intense emotional expression and lower levels of reported emotions in response to emotional imagery, slides, and films compared to nondepressed controls (e.g., Gehricke \& Shapiro, 2000; Rottenberg, Kasch, Gross, \& Gotlib, 2002). This pattern holds for negative and positive emotions and is more robust for the latter (Bylsma, Morris, \& Rottenberg, 2008). Dampening of 
emotional reactivity during depression has been interpreted as a universal feature of this syndrome-evidence of a reduced ability to engage with elicitors of emotions or to generate context-appropriate emotions.

There has long been speculation, however, as to whether these symptoms and the responses to them are shaped by cultural context. For example, Meehl (1975) speculated that Euro-Americans experiencing anhedonia may "develop a kind of 'secondary guilt' or shame about hedonic defects" (p. 305). In the Euro-American cultural context, scripts for healthy emotional functioning involve openly expressing emotions, particularly positive emotions (Bellah, Madsen, Sullivan, Swindler, \& Tipton, 1985; Eid \& Diener, 2001; Wierzbicka, 1999). Dampened emotional reactivity associated with depression may therefore be related to reduced attention to cultural norms regarding emotions; depression may also compromise the ability to enact these norms. Moreover, a failure to experience and express emotions in culturally normative ways may contribute to interpersonal tension and dissatisfaction and over time contribute to maintenance of depressed mood (Ryder \& Chentsova-Dutton, in press). This latter possibility is consistent with the stress generation hypothesis.

It is possible, therefore, that depressed individuals may respond emotionally in ways that are abnormal rela tive to the norms regarding emotions that are empha sized in their cultural contexts. In contrast to European American cultural context, Chinese cultural contexts emphasize emotional moderation and control based on relational demands (Bond, 1991; Russell \& Yik, 1996). Studies comparing emotional reactivity of people from European American and Chinese American cultural con texts suggest that variations in emotional reactivity are consistent with ethnographic accounts of cultural norms regarding emotions. These norms are both widely shared in their contexts and encoded in normative patterns of emotional experience and expression (Eid \& Diener, 2001; Tsai \& Levenson, 1997).

If the cultural norm hypothesis is correct, depressed individuals from Chinese cultural contexts should respond emotionally in ways that are abnormal relative to their cultural context. If so, they would show intensified rather than dampened levels of emotional responding. In support of this hypothesis, studies have found that depression was associated with diminished emotional responding to both negative and positive emotional films in Euro-American samples; Asian American (primarily Chinese American) samples show enhanced or unchanged emotional responding (Chentsova-Dutton et al., 2007; Chentsova-Dutton, Tsai, \& Gotlib, 2010). One notable limitation of this research is that it is cross sectional. As such, it neglects to examine the mechanisms by which failure to adhere to cultural norms regarding emotions and mood influence each other. A developmentally sensitive longitudinal investigation is critical to learning more about the ways that culture and depression shape one another. This work also awaits extension to Chinese majority contexts.

Because people are aware of culturally normative or ideal emotions (Eid \& Diener, 2001; Tsai, Knutson, \& Fung, 2006), the deviations associated with depression may vary in terms of how much they impact subjective well-being. For example, cultural contexts vary in terms of the value placed on experiencing pleasure, with European American contexts emphasizing the importance of pleasure much more than East Asian contexts (Licht, Goldschmidt, \& Schwartz, 2007). A recent momentary sampling study found that anhedonia was associated with reduced ability to feel satisfied with both daily life events and a global evaluation of one's overall life circumstances in European Americans but not Asian Americans (Chentsova-Dutton, Choi, Ryder, \& Reyes, 2011). Generally adhering to normative cultural scripts infuses life with a sense of greater meaning, with positive effects for health (Soto, Chentsova-Dutton, and Lee, in press); failure to do so could generate additional stresses, increasing risk for depression or worsening it. The chaos and confusion of profound distress requires separate interpretive frameworks, dedicated scripts for when things go wrong.

\section{Interpretive Frameworks for Distress}

Given that culture shapes both self-concept and cognitive styles, in turn influencing implicit theories of mind and behavior, it is not surprising that people living in different cultural contexts vary in their preference for differ ent available frameworks to explain distress. According to Haslam's (2005) theory of "folk psychiatry," people process information about abnormality using explanatory frameworks. Several of these frameworks have been described, each with a distinct cognitive underpinning (Haslam, Ban, \& Kaufmann, 2007). The first of these frameworks, pathologizing, is triggered when a normative expectation has been violated. Once pathologized, the behavior is submitted to one or more of three further frameworks: (a) medicalizing, where pathology is attributed to a biological aberration beyond the sufferer's conscious control; (b) moralizing, where pathology is attributed to the sufferer's poor character and conscious decision making; and (c) psychologizing, where pathology is attributed to factors that are internal to the sufferer but beyond their conscious control.

The ways in which these frameworks are taken up depend on local norms, values, and person conceptions. There is evidence, for example, that understanding the reasons why a potentially pathological behavior took place makes it less likely to be pathologized in a Western context but more likely to be pathologized in an East Asian context (Ban, 2008). On a questionnaire, Chinese Singaporean students showed a stronger preference for moralizing explanations of various forms of psychopathology, including depression, whereas 
Euro-Australians showed a stronger preference for psychologizing explanations (Ban, Haslam, \& Kashima, 2010). EuroAustralians live in a cultural context in which psychological explanations help to restore a sense of order and comprehensibility to apparently pathological behaviours. In contrast, Chinese Singaporeans live in a cultural context in which emotional maturity is equated with successful adjustment of behavior to situational demands (Kirmayer, 2007).

Pathologized behaviors, such as emotional expressions that fail to conform to normative cultural scripts, demand explanation if not normalization. Returning to the idea of intersubjectivity (Chiu et al., 2010), sufferers will likely have some insight into the frame others are using to understand their behaviors, and may indeed use that frame themselves. Symptoms are not experienced in isolation but rather with an audience of real and imagined others (Ryder et al., in press). Provided that the social world remains relatively stable, we can use knowledge of a cultural context to make informed predictions about variations in symptoms, emotions, explanatory frames, and so on. Over the longer term, however, there is no reason to assume stability. Increases in individualism and its narcissistic consequences, the importance of positive affect, and the tendency to use psychologized modes of explanation have been documented in "Western" settings, especially the United States (Bellah et al., 1985; Kotchemidova, 2005; Twenge, 2008). Even more dramatically, rapid social change in China could be exerting similarly profound effects in an even shorter period (Kleinman, Yan, Jun, Lee, \& Zhang, 2011). One consequence is the opening up of a rich domain of inquiry, one that demands both developmental and cultural perspectives.

\section{Cultural Development and Change}

We have already observed how rapid change was invoked as a potential explanation for the utility of the stress generation hypothesis in Chinese adolescents. Let us return now to the challenging question of whether cultural change is accompanied by changes in basic psychological processes, applied now to the adult literature. "Challenging" can be understood here both as "difficult to study" and potentially "difficult to accept" if true, especially from the perspective of mainstream psychology. The psychological view of science tacitly assumes that the core phenomena under study are fixed, part of an unchanging human condition. Yet if culture shapes more than clothing, beliefs, food preferences, and so on-and there is mounting evidence that motivation, emotion, and cognition are so shaped-there is little reason to assume that this evidence does not in turn point to the possibility of psychological change over the course of, and in response to, historical events. How might this play out in the rapidly changing $\mathrm{PRC}$ ?

Kleinman (1995) argued that the climate of communist China, especially during the Cultural Revolution, rendered particular emotional experiences and particular diagnoses politically dangerous. Cheung (1991) noted that "during the Cultural Revolution it was considered ideologically undesirable to be depressed" (p. 489); core symptoms of depression could be criticized as decadent individualism, laziness, malingering, or failure to be sufficiently proletarian in outlook (Lee, 1999). Neurasthenia served the function of providing a physical explanation for psychosocial distress, being readily understood at the time as being a product of brain dysfunction (Lee, 1999). A field study conducted in rural China (Cheung, 1989) found that respondents were unwilling to talk about depression but had many examples of neurasthenia, including examples from their own life stories. In this conception, neurasthenia is the way that the body expresses distress that cannot be expressed in other ways (Kleinman, 1995).

When we accept that both cultural contexts and symptom patterns can shift over time, we can no longer assume that the connections we are studying now will persist. Kleinman (1986) followed up on his original study and described striking changes over half a decade. A number of studies now demonstrate that modern concerns revolve around love and money rather than political conformity and party membership (for a review, see Lee, 1999). The Cultural Revolution delegitimized the emotional experiences central to depression; ongoing reforms allow greater expression of these experiences. If the cultural context can shape symptom presentation, cultural change should be expected to bring about symptom change. Symptom change includes strategic differences in what sufferers choose to talk about but also involves deep changes in symptom experience and expression. Indeed, we have argued that a sustained need to engage in a given strategy may be quite conscious at first but is increasingly automatized over time (Ryder \& Chentsova-Dutton, in press).

Different contexts at different times vary in the meaning associated with particular clusters of symptoms, how they are experienced, and how they are presented to others. Simultaneously, professional practice changes in response both to evidence and fashion, in a process that shapes and is shaped by the prevailing cultural climate. Further complicating matters, the concerns of "Western" societies have a pervasive influence on the rest of the world, especially as travel and communication grow more efficient (Kirmayer, 2006). Culture is a moving target; in consequence, so is mental health. The cultural psychology approach leaves plenty of room for change and flux over time, but developmental psychologists have developed numerous tools to study this rigorously. Conversely, the developmental psychology approach leaves plenty of room for the pervasive influence of cultural context, but cultural psychologists have developed numerous tools to study this rigorously. Acceptance that culture and mental health are both moving targets demands, at 
a minimum, sustained collaboration between developmental psychopathology and cultural-clinical psychology.

\section{Developmental Psychopathology and Cultural-Clinical Psychology}

In dividing the literature on depression in China into developmental and cultural research, we have also separated child and adult research, student and patient research, and vulnerability-stress and symptom presentation research. These secondary divisions reflect the particular concerns of the research teams working within these traditions rather than characteristics of the traditions themselves, but they do afford us an opportunity to speculate on what greater integration might look like. What would we learn if we expanded longitudinal research on cognitive vulnerabilities to include direct cross-national comparisons, measuring potential explana tory variables to better help us understand how cultural processes yield different sex ratios? What about testing whether group differences in the presentation of somatic and psychological symptoms emerge at a particular stage of adolescent development, and if so, why this might be? Our argument is not merely that there should be more communication across these disciplines-although that is surely the case—but that researchers in the developmental and cultural traditions can work together to answer questions that might not even be asked otherwise.

Heine and Norenzayan (2006) described two phases of research on cultural difference. In the first phase, cultural group differences are observed and catalogued; in the second, these differences are explained. Explanation proceeds through some variation of what is often called "unpackaging culture," which always requires detailed cultural knowledge of the context or contexts in question and usually includes experimental manipulation, identification of mediators, or some other approach to identifying the specific elements that drive observed group differences. At present, however, there is not much work in the developmental psychopathology literature on depression in China to take us beyond identification of differences when present and attributing those differences to "culture."

The lack of sex difference for cognitive vulnerabilities and depression is one example of an unexpected and potentially important finding that deserves further expli cation. Are there important differences in how girls are socialized in China as compared with "Western" contexts; or, conversely, are there particular pressures on Chinese boys? Do collectivistic value systems provide greater protection to Chinese adolescents from interpersonal stressors by embedding them in social contexts that are not up for constant negotiation and potential loss, while creating greater risk from academic stressors by extending academic failure to loss of face for the entire family? Greater attention to cultural-clinical psychology encourages these sorts of questions and introduces methods by which they can start to be addressed.

In a similar manner, we should consider the ways in which ongoing research on symptom presentation, emotional expressivity, explanatory models, and so on, might benefit from a developmental approach. The most basic ways in which this could be done involve consideration of age cohort effects and the possibility of change over time. For example, given a general tendency for depressed children to emphasize somatic symptoms even in "Western" contexts, what are the processes by which an emphasis on psychological symptoms emerges in some people, in some cultural contexts, but not in others? The cultural norm hypothesis does not currently specify a direction of effect-it may be that depression renders the person less able to express emotions in culturally appropriate ways, but it may also be that people whose style of emotional expression differs from local norms are at increased risk for depression. Longitudinal research designs can help to tease apart these different interpretations.

At present, acknowledgments by one research tradition of the possibilities offered by the other are fleeting, most often in the form of post hoc rationalizations. Findings from Abela and colleagues' research are often explained in the context of rapid modernization and the consequences of the vast changes under way in the PRC. These discussions tend to center around the premise that when the "East" meets the "West," collectivistic cultural values are shifting as the younger generation embraces more individualistic and materialistic values (Auerbach, Abela, et al., 2010; Auerbach, Eberhart, et al., 2010). As a result, traditional familial and social support structures are disrupted, leading to elevated levels of stress and depression. Although discussing these sorts of possibilities is a good first step toward thinking about cultural influence, measuring these constructs would allow their potential explanatory role to be evaluated directly.

Researchers studying adult diagnostic rates and symptom presentation differences have similarly invoked rapid cultural change, from the Cultural Revolution to the current era, to explain changes in the patterns observed over time. Indeed, there is emerging evidence that the tendency for Chinese psychiatric patients to emphasize somatic over psychological symptoms has lessened over a short period of only 6 years (Ryder, 2010). Although such changes may threaten cherished ideas about either diagnoses or "cultures" as more or less fixed, they fit well with ideas both of developmental change and cultures in flux. Indeed, if culture can deeply shape the presentation of psychopathology, it follows that we must accept the possibility of historical shaping as well.

Taking this perspective, however, does not help us to explain exactly how these processes work. As the cultural environment changes, are people being changed along with it so that, for example, someone who would tend to present somatic symptoms 10 years ago is more likely to present psychological symptoms today? Or do these patterns get largely 
fixed by a certain age, so instead what we have is the aging out of cohorts shaped largely by the emotional norms of the Cultural Revolution, and the aging in of new cohorts who came of age during the 1980s and 1990s? Developmental psychopathologists have the tools required to rigorously address such questions; addressing them could teach us much about how emotions are socialized over the lifespan.

The potential benefits, conceptual and practical, that this sort of integration could bring to the study of depression in China lead us to speculate more generally on what an ongoing dialogue between developmental psychopathology and cultural-clinical psychology could bring. Developmental psychopathology has much room in its perspective for the idea that the local context, especially the social context, is closely involved in shaping psychological processes as these develop. Moreover, there is a developmental language with a long tradition that can be drawn upon to describe how this takes place while acknowledging the critical role of the normal maturational processes in the changing organism-its genes, its brain, its body. What cultural and cultural clinical psychology offer are ways to think about the specific influences of specific cultural contexts, a database demonstrating just how fundamental these influences can be, and specific techniques to not just identify cultural differences but unpack processes of cultural shaping.

Cultural-clinical psychology, in a similar manner, contains within it much room for thinking about cultural contexts and cultural identities in flux, changing over time rather than fixed. If anything, there has been a move in this direction in recent years by many cultural psychologists, borrowing and adapting from anthropology. Yet there are few studies in this tradition that actually operationalize a view of culture as change processes. Developmental psychopathology, and developmental psychology more generally, offer ways of thinking about such processes and-perhaps more important, for cultural researchers-specific methods for dealing with them. Arguably the very notion of mutual constitution, so central to cultural psychologists, does not really make sense in the absence of a developmental perspective on how this takes place over time.

\section{Epilogue}

We return in closing to our two scientists and their fish: the first, following the (developmental) progression of the fish down the river; the second, focusing on the (cultural) context at a particular bend. Each agreeing that, of course, the purview of the other is potentially important but remaining focused on a familiar approach. One day, however, they meet each other and begin to talk, and the more they talk, the more they realize not only the importance of the work that is being done by the other, but also how much important work is not being done by either of them, work that needs both approaches in combination to make any sense. They begin to think that there is a need, at least some of the time, to zoom out and take in the whole system. They consider that knowing more about the different local ecologies as the fish progress is important, or that knowing what happens upstream can help in understanding what happens downstream, sometimes in nonobvious ways. They may even realize that a third colleague needs to be brought in to understand how this year's spawning season differs from the norm 10 years ago, or 100 years ago. They may well conclude that this broader perspective is best done in collaboration and not to the exclusion of well-established and narrower approaches. But even as they return to what they do best and resume their work, they do so with new questions and new possibilities in mind.

\section{References}

Abela, J. R. Z., Stolow, D., Mineka, S., Yao, S., Zhu, X., \& Hankin, B. L. (2011). Cognitive vulnerabilities to depressive symptoms in adolescents in urban and rural Hunan, China: A multiwave longitudinal study. Journal of Abnormal Psychology, 120, 765-778.

Abramson, L. Y., Metalsky, G. I., \& Alloy, L. B. (1989). Hopelessness depression: A theory-based subtype of depression. Psychological Review, 96, 358-372.

American Psychiatric Association. (2000). Diagnostic and statistical manual of mental disorders (4th ed., text rev.). Washington, DC: Author.

Auerbach, R. P., Abela, J. R. Z., Zhu, X., \& Yao, S. (2010). Under standing the role of coping in the development of depressive symptoms: Symptom specificity, gender differences, and cross-cultural applicability. British Journal of Clinical Psychology, 49, 547-561.

Auerbach, R. P., Eberhart, N. K., \& Abela, J. R. Z. (2010). Cognitive vulnerability to depression in Canadian and Chinese adolescents. Journal of Abnormal Child Psychology, 38, 57-68.

Bagby, R. M., Ryder, A. G., Schuller, D. R., \& Marshall, M. (2004). The Hamilton Rating Scale for depression: Has the gold standard become a lead weight? American Journal of Psychiatry, 161, 2163-2177.

Ban, L. M. (2008). Pathways to Pathologising: Does understanding behaviour make it seem normal? Unpublished Doctoral Dissertation, University of Melbourne.

Ban, L. M., Haslam, N., \& Kashima, Y. (2010). Does understanding behavior make it seem normal? perceptions of abnormality among Euro-Australians and ChineseSingaporeans. Journal of Cross-Cultural Psychology. Advanced online publication. doi: $10.1177=0022022110385233$

Beck, A. T. (1983). Cognitive therapy of depression: New perspectives. In P. J. Clayton \& J. E. Barrett (Eds.), Treatment of depression: Old controversies and new approaches (pp. 265-284). New York, NY: Raven Press. 
Bellah, R. N., Madsen, R. P., Sullivan, W. M., Swidler, A., \& Tipton, S. M. (1985). Habits of the heart. Berkeley: University of California Press.

Betancourt, H., \& López, S. R. (1993). The study of culture, ethnicity, and race in American psychology. American Psychologist, 48, 629-637.

Bond, M. H. (1991). Beyond the Chinese face. New York, NY: Oxford University Press.

Bruner, J. (1990). Acts of meaning. Cambridge, MA: Harvard University Press.

Bylsma, L. M., Morris, B. H., \& Rottenberg, J. (2008). A meta-analysis of emotional reactivity in major depressive disorder. Clinical Psychology Review, 28, 676-691.

Chen, X., Rubin, K. H., \& Li, B.-S. (1995). Depressed mood in Chinese children: Relations with school performance and family environment. Journal of Consulting and Clinical Psychology, 63, 938-947.

Cheng, C. (1998). Getting the right kind of support: Functional differences in the types of social support on depression for Chinese adolescents. Journal of Clinical Psychology, 54, 845-849.

Chentsova-Dutton, Y. E., Choi, E., Ryder, A. G., \& Reyes, J. (2011). Cultural variation in the effects of anhedonia on well-being. Unpublished manuscript.

Chentsova-Dutton, Y. E., Chu, J. P., Tsai, J. L., Rottenberg, J., Gross, J., \& Gotlib, I. H. (2007). Depression and emotional reactivity: Variation among Asian Americans and European Americans. Journal of Abnormal Psychology, 116, 776-785.

Chentsova-Dutton, Y. E., Tsai, J. L., \& Gotlib, I. (2010). Further evidence for the cultural norm hypothesis: Positive emotion in depressed and control European Americans and Asian Americans. Cultural Diversity and Ethnic Minority Psychology, 16, 284-295.

Cheung, F. M. (1984). Preferences in help-seeking among Chinese students. Culture, Medicine and Psychiatry, 8, 371-380.

Cheung, F. M. (1989). The indigenization of neurasthenia in Hong Kong. Culture, Medicine and Psychiatry, 13, 227-241.

Cheung, F. M. (1991). Health psychology in Chinese societies in Asia. In M. Jansen \& J. Weinman (Eds.), The international development of health psychology (pp. 63-74). Readings, UK: Harwood Academic Press.

Cheung, F. M. (1995). Facts and myths about somatization among the Chinese. In T.-Y. Lin, W. S. Tseng, \& E. K. Yeh (Eds.), Chinese societies and mental health (pp. 156-180). Hong Kong: Oxford University Press.

Chiao, J. Y. (2009). Cultural neuroscience: A once and future discipline. Progress in Brain Research, 178, 287-304.

Chiu, C. Y., Gelfand, M. J., Yamagishi, T., Shteynberg, G., \& Wan, C. (2010). Intersubjective culture: The role of intersubjective perceptions in cross-cultural research. Per- spectives on Psychological Science, 5, 482-493.

Cicchetti, D., Rogosch, F. A., Gunnar, M. R., \& Toth, S. L. (2010). The differential impacts of early abuse on internalizing problems and daytime cortisol rhythm in school-aged children. Child Development, 81, 252-269.

Connor-Smith, J. K., \& Compas, B. E. (2004). Coping as a moderator of relations between reactivity to interpersonal stress, health status, and internalizing problems. Cognitive Therapy and Research, 28, 347-368.

Craig, T. K., \& Boardman, A. P. (1990). Somatization in primary care settings. In C. M. Bass (Ed.), Physical symptoms and psychological illness (pp. 73-103). Oxford, UK: Blackwell Scientific Publications.

Dere, J., Tang, Q., Zhu, X., Cai, L., Yao, S., \& Ryder, A. G. (2011). The cultural shaping of alexithymia: Values and externally oriented thinking in a Chinese clinical sample. Unpublished manuscript.

Dere, J., Falk, C. F., \& Ryder, A. G. (2012). Unpacking cultural differences in alexithymia: The role of cultural values among Euro-Canadian and Chinese-Canadian students. Journal of Cross Cultural Psychology. Advance online publication. Retrieved January 31, 2012. DOI: $10.1177=0022022111430254$.

DiMaggio, P. (1997). Culture and cognition. Annual Review of Sociology, 23, 263-287.

Dion, K. L. (1996). Ethnolinguistic correlates of alexithymia: Toward a cultural perspective. Journal of Psychosomatic Research, 41, 531-539.

Draguns, J. G. (1996). Abnormal behaviour in Chinese societies: Clinical, epidemiological, and comparative studies. In M. H. Bond (Ed.), Handbook of Chinese psychology (pp. 412-428). Oxford, UK: Oxford University Press.

Eberhart, N. K., \& Hammen, C. L. (2009). Interpersonal predictors of stress generation. Personality and Social Psychology Bulletin, 35, 544-556.

Eid, M., \& Diener, E. (2001). Norms for experiencing emotions in different cultures: Inter- and intranational differences. Journal of Personality and Social Psychology, 81, 869-885.

Ellis, B. J., Shirtcliff, E. A., Boyce, W. T., Deardorffm J., \& Essex, M. J. (2011). Quality of early family relationships and the timing and tempo of puberty: Effects depend on biological sensitivity to context. Development and Psychopathology, 23, 85-99.

Flynn, M., Kecmanovic, J., \& Alloy, L. (2010). An examination of integrated cognitive-interpersonal vulnerability to depression: The role of rumination, perceived social support, and interpersonal stress generation. Cognitive Therapy and Research, 34, 456-466.

Gehricke, J., \& Shapiro, D. (2000). Reduced facial expression and social context in major depression: Discrepancies between facial muscle activity and self-reported emotion. Psychiatry Research, 95, 157-167. 
Gibb, B. E., \& Hankin, B. L. (2010). In memoriam: John R. Z. Abela. International Journal of Cognitive Therapy, 3, 211-212.

Grant, K. E., Compas, B. E., Thurm, A. E., McMahon, S. D., Gipson, P. Y., Campbell, A. J., ... Westerholm, R. I. (2006). Stressors and child and adolescent psychopathology: Evidence of moderating and mediating effects. Clinical Psychology Review, 26, 257-283.

Greenberger, E., Chen, C., Tally, S. R., \& Dong, Q. (2000). Family, peer, and individual correlates of depressive symptomatology among U.S. and Chinese adolescents. Journal of Consulting and Clinical Psychology, 68, 209-219.

Hammen, C. (1991). Generation of stress in the course of unipolar depression. Journal of Abnormal Psychology, 100, 555-561.

Hankin, B. L. (2006). Adolescent depression: Description, causes, and interventions. Epilepsy $\mathcal{F}$ Behavior, 8, 102-114.

Hankin, B. L. (2008). Stability of cognitive vulnerabilities to depression: A short-term prospective multi-wave study. Journal of Abnormal Psychology, 117, 324-333.

Hankin, B. L., Badanes, L. S., Abela, J. R. Z., \& Watamura, S. E. (2010). Hypothalamic- Pituitary- Adrenal Axis dysregulation in dysphoric children and adolescents: Cortisol reactivity to psychosocial stress from preschool through middle adolescence. Biological Psychiatry, 68, 484-490.

Haslam, N. (2005). Dimensions of folk psychiatry. Review of General Psychology, 9, 35-47.

Haslam, N., Ban, L., \& Kaufmann, L. (2007). Lay conceptions of mental disorder: The folk psychiatry model. Australian Psychologist, 42, 129-137.

Heine, S. J., \& Norenzayan, A. (2006). Toward a psychological science for a cultural species. Perspectives for Psychological Science, 1, 251-269.

Hesketh, T., \& Ding, Q. J. (2005). Anxiety and depression in adolescents in urban and rural China. Psychological Reports, 96, 435-444.

Hesketh, T., Ding, Q. J., \& Jenkins, R. (2002). Suicide ideation in Chinese adolescents. Social Psychiatry and Psychiatric Epidemiology, 37, 230-235.

Hong, W., Abela, J. R. Z., Cohen, J. R., Sheshko, D. M., Shi, X. T., Hamel, A. V., \& Starrs, C. (2010). Rumination as a vulnerability fac tor to depression in adolescents in Mainland China: Lifetime history of clinically significant depressive episodes. Journal of Clinical Child $\mathcal{E}$ Adolescent Psychology, 39, 849-857.

Hwang, W.-C., \& Myers, H. (2007). Major depression in Chinese Americans. Social Psychiatry and Psychiatric Epidemiology, 42, 189-197.

Hwu, H.-G., Yeh, E.-K., \& Chang, L.-Y. (1989). Prevalence of psychiatric disorders in Taiwan defined by the Chinese Diagnostic Interview Schedule. Acta Psychiatrica Scandinavica, 79, 136-147.
Kashima, Y. (2000). Conceptions of culture and person for psychology. Journal of Cross-Cultural Psychology, 31, 14-32.

Kessler, R. C., McGonagle, K. A., Zhao, S., Nelson, C. B., Hughes, M., Eshleman, S., ... Kendler, K. S. (1994). Lifetime and 12-month prevalence of DSM-III-R psychiatric disorders in the United States: Results from the National Comorbidity Survey. Archives of General Psychiatry, 51, 8-19.

Kirmayer, L. J. (1987). Languages of suffering and healing: Alexithymia as a social and cultural process. Transcultural Psychiatric Research Review, 24, 119-136.

Kirmayer, L. J. (2001). Cultural variations in the clinical presentation of depression and anxiety: Implications for diagnosis and treatment. Journal of Clinical Psychiatry, 62(Suppl. 13), 22-28.

Kirmayer, L. J. (2006). Beyond the "new cross-cultural psychiatry": Cultural biology, discursive psychology and the ironies of globalization. Transcultural Psychiatry, 43, 126-144.

Kirmayer, L. J. (2007). Psychotherapy and the cultural concept of the person. Transcultural Psychiatry, 44, 232-257.

Kirmayer, L. J. (2012). The future of critical neuroscience. In S. Choudhury \& J. Slaby (Eds.), Critical Neuroscience: A Handbook of the Social and Cultural Contexts of Neuroscience (pp. 367-383). Oxford: Blackwell.

Kitayama, S., \& Uskul, A. K. (2011). Culture, mind, and the brain: Current evidence and future directions. Annual Review of Psychology, 62, 419-449.

Kleinman, A. (1982). Neurasthenia and depression: A study of somatization and culture in China. Culture, Medicine, and Psychiatry, 6, 117-190.

Kleinman, A. (1986). Social origins of disease and distress: Depression, neurasthenia, and pain in modern China. New Haven, CT: Yale University Press.

Kleinman, A. (1995). Writing at the margin: Discourse between anthro pology and medicine. Berkeley: University of California Press.

Kleinman, A., Yan, Y., Jun, J., Lee, S., \& Zhang, E. (2011). Deep China: The moral life of the person. Berkeley: University of California Press.

Kotchemidova, C. (2005). From good cheer to "drive-by smiling": A social history of cheerfulness. Journal of Social History, 39, 5-37.

Lee, S. (1999). Diagnosis postponed: Shenjing Shuairuo and the trans formation of psychiatry in post-Mao China. Culture, Medicine and Psychiatry, 23, 349-380.

Leung, P. W. L., \& Wong, M. M. T. (1998). Can cognitive distortions differentiate between internalising and externalising problems? Journal of Child Psychology and Psychiatry, 39, 263-269.

Li, H., \& Zhang, Y. (2008). Factors predicting rural Chi- 
nese adolescents' anxieties, fears and depression. School Psychology International, 29, 376-384.

Licht, A. N., Goldschmidt, C., \& Schwartz, S. H. (2007). Culture rules: The foundations of the rule of law and other norms of governance. Journal of Comparative Economics, 35, 659-688.

Liu, X. C., Ma, D. D., Kurita, H., \& Tang, M. Q. (1999). Self-reported depressive symptoms among Chinese adolescents. Social Psychiatry $\mathcal{F}$ Psychiatric Epidemiology, 34, 44-47.

Magaro, M., \& Weisz, J. (2006). Perceived control mediates the relation between parental rejection and youth depression. Journal of Abnormal Child Psychology, 34, 863-872.

Markus, H. R, \& Kitayama, S. (1991). Culture and the self: Implications for cognition, emotion, and motivation. Psychological Review, 98, 224-253.

Meehl, P. (1975). Hedonic capacity: Some conjectures. Bulletin of the Menninger Clinic, 39, 295-307.

Murray, C. J. L., \& López, A. D. (1996). The global burden of disease (Vol. 1). Boston, MA: Harvard School of Public Health.

Ng, C. S. M., \& Hurry, J. (2011). Depression amongst Chinese adolescents in Hong Kong: An evaluation of a stress moderation model. Social Indicators Research, 100, 499-516.

Nolen-Hoeksema, S. (2000). The role of rumination in depressive disorders and mixed anxiety=depressive symptoms. Journal of Abnormal Psychology, 109, 504-511.

Park, R. J., Goodyear, I. M., \& Teasdale, J. D. (2004). Effects of induced rumination and distraction on mood and overgeneral auto biographical memory in adolescent Major Depressive Disorder and controls. Journal of Child Psychology and Psychiatry, 45, 996-1006.

Parker, G., Cheah, Y. C., \& Roy, K. (2001). Do the Chinese somatize depression? A cross-cultural study. Social Psychiatry and Psychiatric Epidemiology, 36, 287-293.

Richerson, P. J., \& Boyd, R. (2005). Not by genes alone: How culture transformed human evolution. Chicago, IL: The University of Chicago Press.

Rottenberg, J., Kasch, K. L., Gross, J. J., \& Gotlib, I. H. (2002). Sadness and amusement reactivity differentially predict concurrent and prospective functioning in major depressive disorder. Emotion, 2, 135-146.

Rudolph, K. D., \& Hammen, C. (1999). Age and gender as determinants of stress exposure, generation, and reactions in youngsters: A transactional perspective. Child Development, 70, 660-677.

Russell, J. A., \& Yik, M. S. M. (1996). Emotion among the Chinese. In M. H. Bond (Ed.), The handbook of Chinese psychology (pp. 166-188). Hong Kong: Oxford University Press.

Ryder, A. G. (2010, November). Modernization, Westernization, and Urbanization: Sociocultural effects on depres- sive symptoms in China and South Korea. Invited talk delivered at the University of Heidelberg, Conference on Intercultural Aspects of Mental Disorders, Heidelberg, Germany.

Ryder, A. G., Ban, L. M., \& Chentsova-Dutton, Y. E. (2011). Towards a cultural-clinical psychology. Social and Personality Psychology Compass, 5, 960-975.

Ryder, A. G., Ban, L. M., \& Dere, J. (in press). Culture, self, and symptom: Perspectives from cultural psychology. In T. Hansen, K. de Lopez, \& P. Berliner (Eds.), Culture in self in mind: Conceptual and applied approaches. Aalborg, Denmark: Aalborg University Press.

Ryder, A. G., \& Chentsova-Dutton, Y. E. (2012). Depression in cultural context: "Chinese somatization", revisted. Psychiatric Clinics of North America, 35, 15-36.

Ryder, A. G., Yang, J., Zhu, X., Yao, S., Yi, J., Heine, S. J., \& Bagby, R. M. (2008). The cultural shaping of depression: Somatic symptoms in China, psychological symptoms in North America? Journal of Abnormal Psychology, 117, 300-313.

Sastry, J., \& Ross, C. E. (1998). Asian ethnicity and the sense of personal control. Social Psychology Quarterly, 61, 101-120.

Shweder, R. A. (1991). Thinking through cultures: Expeditions in cultural psychology. Cambridge, MA: Harvard University Press.

Soto, J. A., Chentsova-Dutton, Y. E., \& Lee, E. A. (in press). The interplay of culture and emotions in maintaining health in the con text of relationships. In M. Newman \& N. Roberts (Eds.), Handbook of health and social relationships. Washington, DC: American Psychological Association.

Starrs, C. J., Abela, J. R. Z., Shih, J. H., Cohen, J. R., Yao, S., Zhu, X. Z., \& Hammen, C. L. (2010). Stress generation and vulnerability in adolescents in mainland China. International Journal of Cognitive Therapy, 3, 345-357.

Stewart, S. M., Kennard, B. D., Lee, P. W. H., Hughes, C. W., Mayes, T. L., Emslie, G. J., \& Lewinsohn, P. M. (2004). A cross-cultural investigation of cognitions and depressive symptoms in adolescents. Journal of Abnormal Psychology, 113, 248-257.

Stewart, S. M., Lewinsohn, P. M., Lee, P. W. H., Ho, L. M., Kennard, B., Hughes, C. W., \& Emslie, G. J. (2002). Symptom patterns in depression and "subthreshold" depression among adolescents in Hong Kong and the United States. Journal of Cross-Cultural Psychology, 33, 559-576.

Sun, Y., Tao, F., Hao, J., \& Wan, Y. (2010). The mediating effects of stress and coping on depression among adolescents in China. Journal of Child and Adolescent Psychiatric Nursing, 23, 173-180.

Tsai, J. L., Knutson, B., \& Fung, H. H. (2006). Cultural variation in affect valuation. Journal of Personality and Social Psychology, 90, 288-307.

Tsai, J. L., \& Levenson, R. W. (1997). Cultural influences of emotional responding: Chinese American and European 
American dating couples during interpersonal conflict. Journal of Cross-Cultural Psychology, 28, 600-625.

Twenge, J. M. (2008). Generation Me, the origins of birth cohort differences in personality traits, and cross-temporal meta-analysis. Social and Personality Psychology Compass, 2, 1440-1454.

Weissman, M. M., Bland, R. C., Canino, G. J., Faravelli, C., Greenwald, S., Hwu, H.-G., ... Yeh, E. K. (1996). Crossnational epidemiology of Major Depression and Bipolar Disorder. The Journal of the American Medical Association, 276, 293-299.

Weisz, J. R., Rothbaum, F., \& Blackburn, T. F. (1984). Standing out and standing in: The psychology of control in the U.S. and Japan. American Psychologist, 39, 955-969.

Wexler, B. E. (2006). Brain and culture: Neurobiology, ideology, and social change. Cambridge, MA: MIT Press.

Wierzbicka, A. (1999). Emotions across languages and cultures: Diversity and universals. New York, NY: Cambridge University Press.

Wu, J. (2006, June 9). Survey highlights youth depression. The China Daily. Retrieved from http://www.chinadaily.com.cn/

Xiao, J., Yao, S., Zhu, X., Zhang, C., Auerbach, R. P., McWhinnie, C. M., \& Abela, J. R. Z. (2010). The responses to stress questionnaire: construct validity and prediction of depressive and social anxiety symptoms in a sample of Chinese adolescents. Stress and Health, 26, 238-249.

Yang, J., Yao, S., Zhu, X., Zhang, C., Ling, Y., Abela, J. R. Z., ... McWhinnie, C. (2010). The impact of stress on depressive symptoms is moderated by social support in Chinese adolescents with sub threshold depression: A multiwave longitudinal study. Journal of Affective Disorders, 127, 113-121.

Yen, S., Robins, C. J., \& Lin, N. (2000). A cross-cultural comparison of depressive symptom manifestation: China and the United States. Journal of Consulting and Clinical Psychology, 68, 993-999.

Zhou, T., Zhang, S., Jiang, Y., \& Wang, J. (2000). Epidemiology of neuroses in a Shanghai community. Chinese Mental Health Journal, 14, 332-334. 